\title{
Shape Classification of Red Blood Cell Image Based on Tetrolet Transform
}

\author{
Ruihu Wang \\ Key Laboratory of Machine Vision and Intelligent Information System, Chongqing University of Arts and Sciences, Chongqing,
}

China

\begin{abstract}
The shape of red blood cell is a primary factor for its deformability and filterability. Generally the regular shape of red blood cells should look like a biconcave disk. Meanwhile the deformability degradation of erythrocyte could lead to some blood-related diseases. Thus the shape analysis of red blood cells will make sense in real application. In this paper, the different shape illustration of red blood cells was briefly introduced at first. And then some preliminary about Tetrominoes was reviewed. In the end we proposed a methodology framework which intends to extract the shape feature with Tetrolet transform.
\end{abstract}

Keywords-shape classification; deformation; red blood cell; tetrolet transform

\section{INTRODUCTION}

Erythrocytes (Red Blood Cells) are created in the bone marrow, and after about 120 days they will degrade in the spleen and liver. They are the most common (>99\%) blood cells and of nucleus and organelles. As we well known, the erythrocytes are the most important to maintain human's normal physiology function. Furthermore, there is considerable evidence proving that erythrocyte deformability is an important determinant in the filterability of blood and consequently in the pathology of a variety of blood-related diseases. In addition, the shape of red blood cell is also a determining factor for its deformability and filterability. As a matter of fact, Myalgic Encephalomyelitis (ME) and Multiple Sclerosis (MS) arise from the deformability degradation of erythrocyte in pathology research. Therefore when clinic diagnosing, the shape analyzing of erythrocyte is helpful for physician to determine what kind of state of an illness the patient suffered.

The most primarily used method for analyzing erythrocytes deformability is extracting the shape feature of various kinds of cells in a red blood cell image. The original input red blood cell image we are aiming to treat with is captured by Scanned Electronic Microscope (SEM). As a matter of fact, the shape of each red blood cell may take on very different kind of formation. As shown in Figure 1, the shape diversity and deformation with different direction brings some challenges to our research.

In recent years, as one of the newly multi-scale geometrical analysis technology, Tetrolet transform has been proposed. It has drawn more consideration in image processing domain and achieved great improvements.

In [1], the authors exploits the advantages of the tensor product wavelet transform for representing smooth images and the ability of the tetrolet transform to represent texture and edge effectively at the same time to get an optimal sparse approximation for remote sensing image. Xu et.al decomposed sample images of hot-rolled steel plates into multiple sub-bands with different scales and directions by Tetrolet transform. The high-pass Tetrolet coefficients of sub-bands are combined into a high dimension feature vector. With KLPP dimensional reduction and SVM classifier, the surface defect of hot-rolled steel plates would be recognized with higher correct recognition rate [2]. Tetrolet transform is able to optimally approximate multiple geometrical characteristics, with which Tian el.al extract spectral texture feature of satellite image successfully in [3].

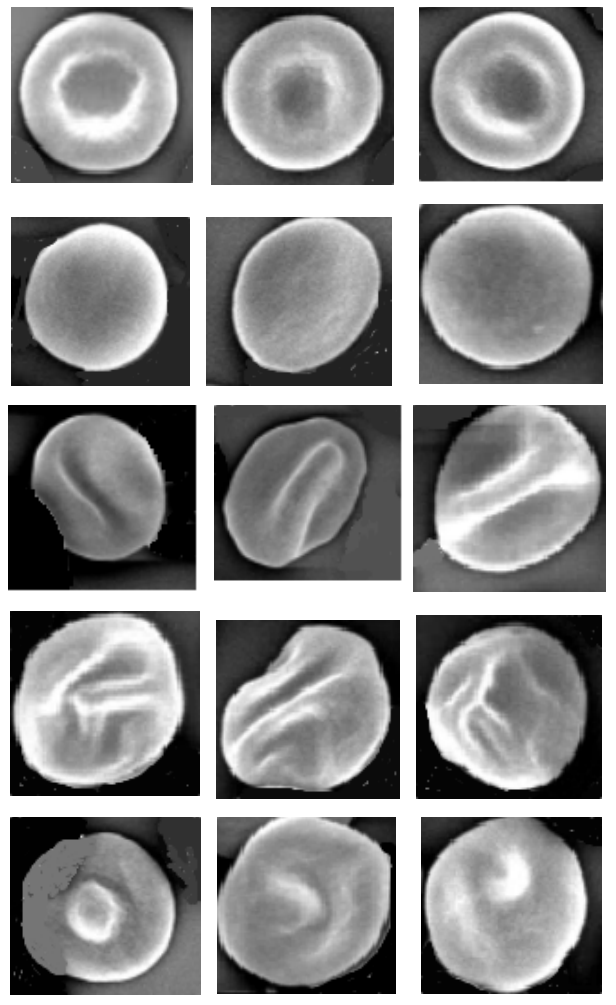

FIGURE I. DIFFERENT SHAPE OF RED BLOOD CELLS

Manoj Kumar et. al presented a new approach to denoise CT image using locally adaptive shrinkage rule in tetrolet domain, the proposed scheme is superior to the existing methods in terms of visual quality [4]. 


\section{Shape Deformation CHARACTERISTIC OF RED BLOOD CELL IMAGE}

Due to the variability of the shape of the red blood cells illustrated in Figure 1, the commonly used classification method does not apply to such special objects. Traditional artificial analysis takes a long time and being with low precision, which depends on the visual impression and other unfavorable factors to a large extent. It is necessary to combine the deformation characteristics of red blood cells with their shapes.

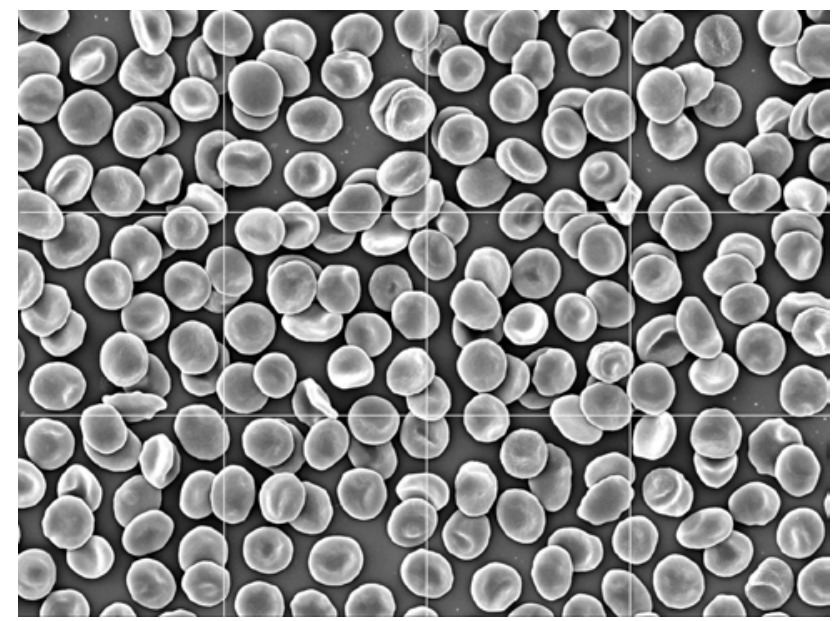

FIGURE II. SEM IMAGE OF RED BLOOD CELL

As there are lots of overlapped red blood cells in the original image shown in Figure 2, we only interested in the top level cells with regarding to statistics feature. Each cell is extracted individually from the whole image by using region growing algorithm which is based on boundary contour tracing. The segmentation algorithm consists of two main procedure, contour tracing and region growing respectively [5].

\section{PRILIMINARY}

For an image $g, g=g(m, n)$, let

$$
I=\{(m, n): m, n=0, \cdots, M-1\} \subset \mathbb{Z}^{2}
$$

is the index set of $g$, where $M=2^{J}, J \in \mathbb{N}$.

The 4-neighborhood $\hat{n}$ of index $(m, n)$ is defined as:

$$
\hat{n}(m, n)=\{(m-1, n),(m+1, n),(m, n-1),(m, n+1)\}
$$

As shown in Figure 3, there are two neighbors for that index lies at the vertex of an image like (b), and three neighbors for that index lies at the boundary as well like (c). Apart from this, there are four neighbors for other indices.

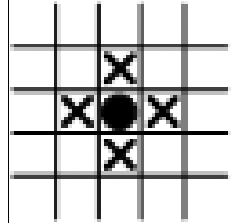

(a)

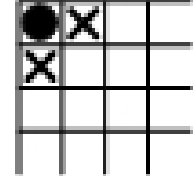

(b)

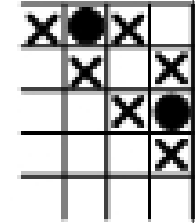

(c)
FIGURE III. (A) 4-NEIGHBORHOOD OF (M,N). (B) TWO NEIGHBORS OF VERTEX INDEX. (C) THREE NEIGHBORS OF BOUNDARY INDEX

The image is divided into different partitions denoted by $I_{u}$, $I_{v}, I_{w}$, etc, as shown in Figure 4, where

$$
I_{u, v, w, \cdots} \subset F=\left\{I_{0}, I_{1}, \cdots, I_{r}\right\}, r \in \mathbb{N} .
$$

And it satisfies two conditions:

1. Each subset $I_{u}$ contains four indices.

2. Every index of $I_{u}$ has a neighbor in $I_{u}$, namely, for each $(m, n) \in I_{u} \quad$, there exists $\left(m^{\prime}, n^{\prime}\right) \in I_{u} \quad$ such that $\left(m^{\prime}, n^{\prime}\right) \in \hat{n}(m, n)$

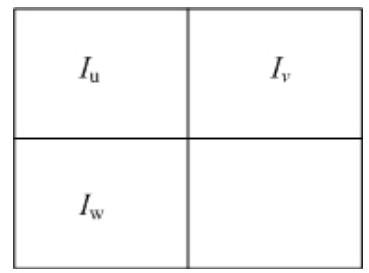

FIGURE IV. IMAGE PARTITION

As shown in Figure 5, although the two subsets $I_{v}$ and $I_{w}$ contain four indices, The three indices labeled by $\times$ do not conform to the second condition. All of them have no 4neighborhood indices.
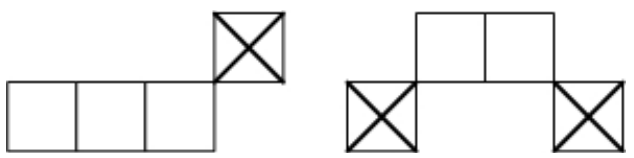

FIGURE V. CONTAINING 4 INDICES BUT NOT SATISFYING THE 2ND CONDITIONS

Such subsets $I_{u}$ are called tetromino. The concept of Tetrominoes was proposed by Golomb, which are some shapes formed by a combination of four equal-sized unit squares [6]. There are five different shapes regardless the rotations and reflections as shown in Figure 6. 


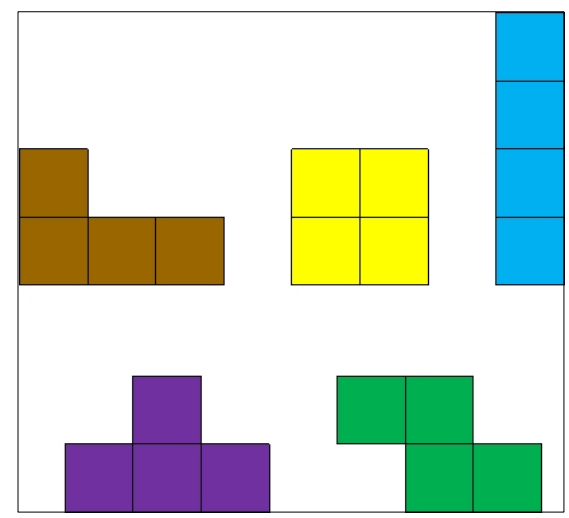

FIGURE VI. THE FIVE FREE TETROMINOES(L-O-I-T-S)

Each square $[0, N)^{2}$ whose size is $N \times N$ can be covered by tetrominoes if and only if $N$ is even. With $N$ increasing, the number of different combinations explodes increasingly. In 1937, Larsson proved that there are 117 solutions together for disjoint covering of a $4 \times 4$ block with 4 tetrominoes [7]. There exists 22 fundamental configurations titling a $4 \times 4$ block (disregarding rotations and reflections), whereas there are 117 solutions if we take the rotations and reflection into account. The 22 fundamental forms are shown in Figure 7 [8].

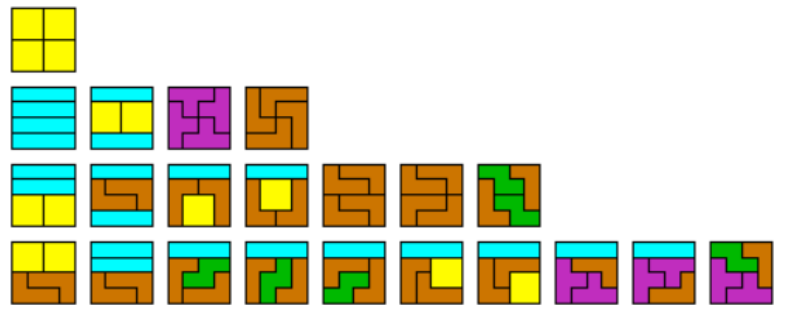

FIGURE VII. THE 22 FUNDAMENTAL CONFIGURATION $(1+4+7+10)$

Figure.8 (a) represents one of the covering from 117 types of titling and (c) show its local structure.

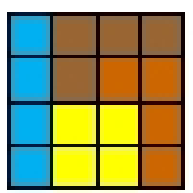

(a)

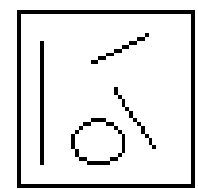

(b)
FIGURE VIII. (A) ONE OF THE DISJOINT COVERING OF 117 TYPES OF TITLINGS. (C)LOCAL STRUCTURE OF (B)

\section{Tetrolet Transform}

In order to obtain an efficient image representation, Jens Krommweh introduced a new adaptive Haar wavelet transform, namely Tetrolet Transform, which is especially designed for sparse image representation. The idea of Tetrolet transform grew out of a famous computer game 'Tetris', in which five geometric models as shown in Figure 6 are combined randomly with rotation and reflection properties [9].

Classical 2D Haar wavelet decomposition can bring about special Tetromino partition, which is based on the design of dividing the whole image block into $2 \times 2$ structure. It is only one type of configuration of total 117 forms. Moreover, the partition generated by Tetrolet transform is more effective, which decompose each $4 \times 4$ block into 4 uncovered tetronimoes in terms of the context and geometrical structure of image.

For a given input image $g$,

$$
g=(g[m, n])_{m, n=0}^{N-1}, N=2^{J}, J \in \mathbb{N}
$$

altogether $J-1$ levels of the tetrolet transform can be implemented on it. In the rth level, $r \in\{1, \cdots, J-1\}$, divide the low-pass part image $a^{r-1}$ into $4 \times 4$ image blocks $Q_{m, n}$.

For each block $Q_{m, n}$, all the possible 117 configurations of the tetromino covering will be performed. The Haar wavelet transform is run on each titling which containing the four tetromimo subsets. After that the low pass and high pass coefficient are computed as:

$$
g^{r,(c)}=\left(g^{r,(c)}[s]\right)_{s=0}^{3} ; w_{l}^{r,(c)}=\left(w_{l}^{r,(c)}[s]\right)_{s=0}^{3},
$$

Where $g^{r,(c)}$ denote the pixel averages of admissible tetronimoes, and $w_{l}^{r,(c)}$ denote the three high pass parts for $l=1,2,3$ respectively.

Then the covering parameter $C^{*}$ is acquired by minimizing the $l^{1}$-norm of the tetrolet coefficients.

$$
c^{*}=\underset{c}{\arg \min } \sum_{l=1}^{3}\left\|w_{l}^{r,(c)}\right\|_{1}=\arg \min \sum_{l=1}^{3} \sum_{s=0}^{3}\left|w_{l}^{r,(c)}[s]\right|
$$

Rearrange the low pass coefficient $a^{r,\left(c^{*}\right)}$ and high pass coefficient $w_{l}^{r,\left(c^{*}\right)}$ into $2 \times 2$ matrices respectively.

$$
\begin{gathered}
g_{\mid Q_{m, n}}^{r}=R\left(g^{r,\left(c^{*}\right)}\right)=\left[\begin{array}{ll}
g^{r,\left(c^{*}\right)}[0] & g^{r,\left(c^{*}\right)}[2] \\
g^{r,\left(c^{*}\right)}[1] & g^{r,\left(c^{*}\right)}[3]
\end{array}\right] \\
w_{l \mid Q_{m, n}}^{r}=R\left(w_{l}^{r,\left(c^{*}\right)}\right)=\left[\begin{array}{ll}
w_{l}^{r,\left(c^{*}\right)}[0] & w_{l}^{r,\left(c^{*}\right)}[2] \\
w_{l}^{r,\left(c^{*}\right)}[1] & w_{l}^{r,\left(c^{*}\right)}[3]
\end{array}\right]
\end{gathered}
$$

After Tetrolet decomposition for each image block, we store corresponding high-pass matrix and tilting parameters $C^{*}$ for feature extraction of high-pass matrix and image reconstruction based on Tetrolet inverse transformation.

\section{FUTURE WORK}

Since lots of improvements have been achieved by using Tetrolet transform for image processing. Additionally the 
shapes of red blood cells are diversified and the deformations are presented with different directions together with multi scale geometrical characteristics, we proposed a methodology framework for red blood cell shape classification as shown in Figure 9.

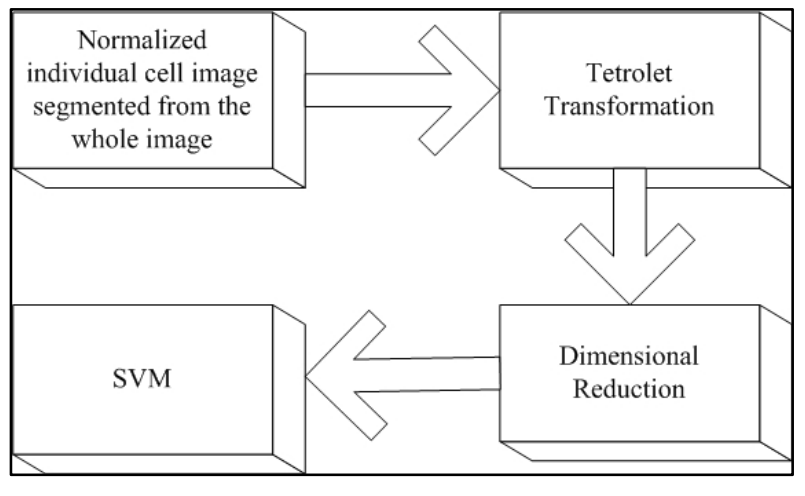

FIGURE IX. METHODOLOGY FRAMEWORK FOR SHAPE CLASSIFICATION

Firstly, the extracted individual cell image segmented from the whole image will be normalized, then Tetrolet transform will be performed on them. After dimensional reduction processing with PCA etc, the extracted tetrolet features will be trained and classified with Support Vector Machine.

\section{ACKNOWLEDGMENT}

This research was financially supported by the Science and Technology Foundations of Chongqing Municipal Education Commission under Grant No.KJ131225, and Scientific and Technological Project of Yongchuan District under (Ycstc2013ad5001).

\section{REFERENCES}

[1] Cuiping Shi, Junping Zhang, Hao Chen, Ye Zhang. 2014. A Novel Hybrid Method for Remote Sensing Image Approximation Using the Tetrolet Transform. IEEE Journal of Selected Topics in Applied Earth Observations and Remote Sensing. 7(12), December 2014:4949-4960.

[2] XU Ke, WANG Lei, WANG Jingyu. 2016. Surface Defect Recognition of Hot-rolled Steel Plates based on Tetrolet Transform. Chinese Journal of Mechanical Engineering. 52(4), Feb 2016:13-19

[3] TIAN Wen-zhe, FU Ran-di, JIN Wei, ZHOU Ying. Research on Cumulonimbus Detection based on Both Tetrolet Transform and Support Vector Machine. Mobile Communication, 2015.12: 72-77

[4] Manoj Kumar, Manoj Diwakar. CT image denoising using locally adaptive shrinkage rule in tetrolet transform. Journal of King Saud University-Computer and Information Sciences (2016), http://dx.doi.org/10.1016/j.jksuci.2016.03.003

[5] R. Wang, B. Wang. A Combined Approach on RBC Image Segmentation Through Shape Feature Extraction. Mathematical Problems in Engineering. 2012.03

[6] S.W.Golomb. 1965. Polyominoes. New York, NY, USA: Scribner, 1965, pp:70-85

[7] B. Larsson, Problem 2623, Fairy Chess Rev. 3 (5) (1937) 51.

[8] Jens Krommweh. 2009. Image Approximation by Adaptive Tetrolet Transform. Laurent Fesquet and Bruno Torresani. SAMPTA’09, May 2009, Marseille, France. Special session on geometric multiscale analysis, 2009

[9] Jens Krommweh. 2010. Tetrolet transform: A new adaptive Haar wavelet algorithm for sparse image representation. Journal of Visual Communication and Image Representation, 2010, 21(4):364-374.J. Clerk
Maxwell, A Treatise on Electricity and Magnetism, 3rd ed., vol. 2. Oxford: Clarendon, 1892, pp.68-73 